\title{
UNILATERAL 3D EXTERNAL FIXATION IN SERBIAN TRAUMATOLOGY AND ORTHOPAEDICS
}

\author{
Sasa Milenkovic ${ }^{1}$, Milan Mitkovic $^{2}$, Milorad Mitkovic $^{3}$, Marko Bumbasirevic $^{4}$, and Vojislav \\ Bumbasirevic ${ }^{5}$ \\ ${ }^{1}$ University of Nis Faculty of Medicine \\ ${ }^{2}$ Clinical Center Nis \\ ${ }^{3}$ University of Nis, Faculty of Medicine \\ ${ }^{4}$ Serbian Academy of Sciences and Arts \\ ${ }^{5}$ University Clinical Center of Serbia
}

April 16, 2021

\begin{abstract}
Objective Although it may have been criticized and questioned by numerous authors, external fixation holds its place in modern orthopaedics and traumatology. The aim of this paper is to show the applicability of the unilateral 3D external fixation in everyday practice. Methods For external fixation of the bones, we used unilateral 3D external fixators according to Mitkovic. Results In the Republic of Serbia, the Mitkovic unilateral 3D external fixation system has been used in over 30,000 cases. The indication field of applicability of this method is wide. External fixation method was mostly used for the treatment of open and closed tibial fractures at all levels, in open femoral fractures as a part of the "staging" protocol and in fractures of the upper limbs. We used external fixation for the treatment of nonunions, malunions, in the treatment of osteitis, septic pseudoarthrosis, angular knee deformities, post-traumatic deformities, for limb lengthening and in replantation surgery. Conclusion The unilateral external fixator enables 3D biomechanical stability and is suitable for use in everyday traumatology and orthopaedics practice.
\end{abstract}

\section{Hosted file}

IJCP.pdf available at https://authorea.com/users/408112/articles/518259-unilateral-3dexternal-fixation-in-serbian-traumatology-and-orthopaedics 

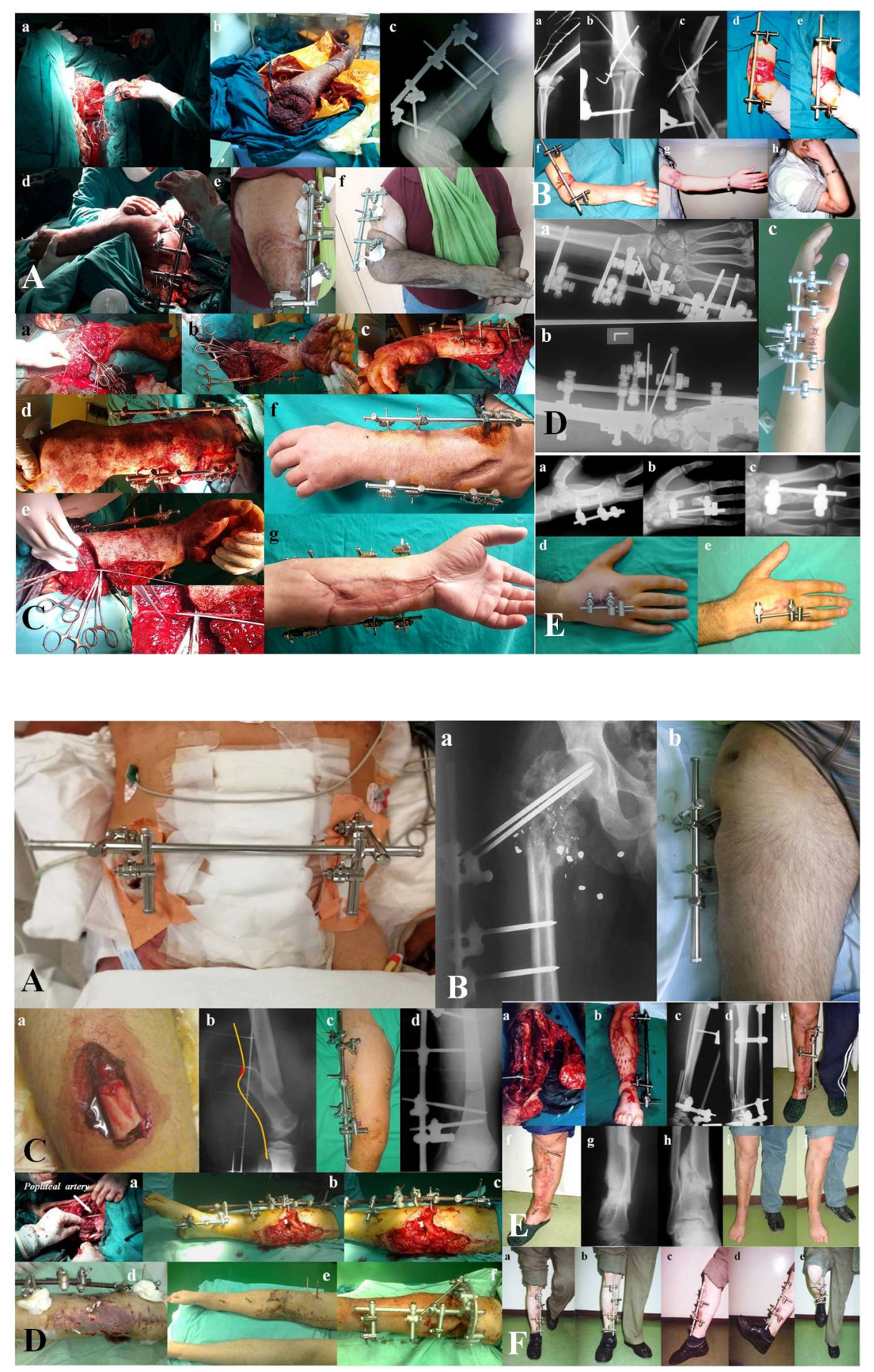


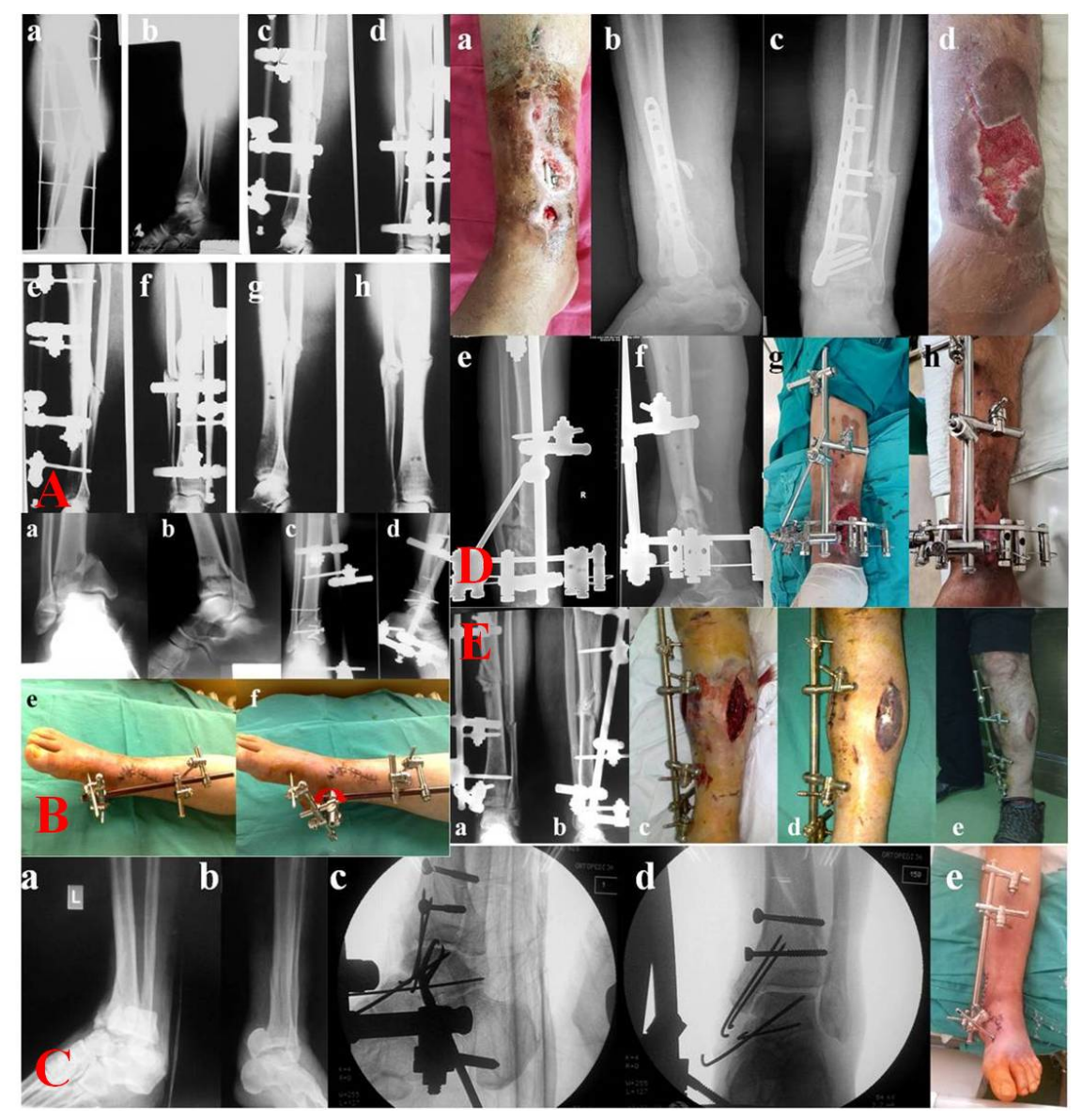



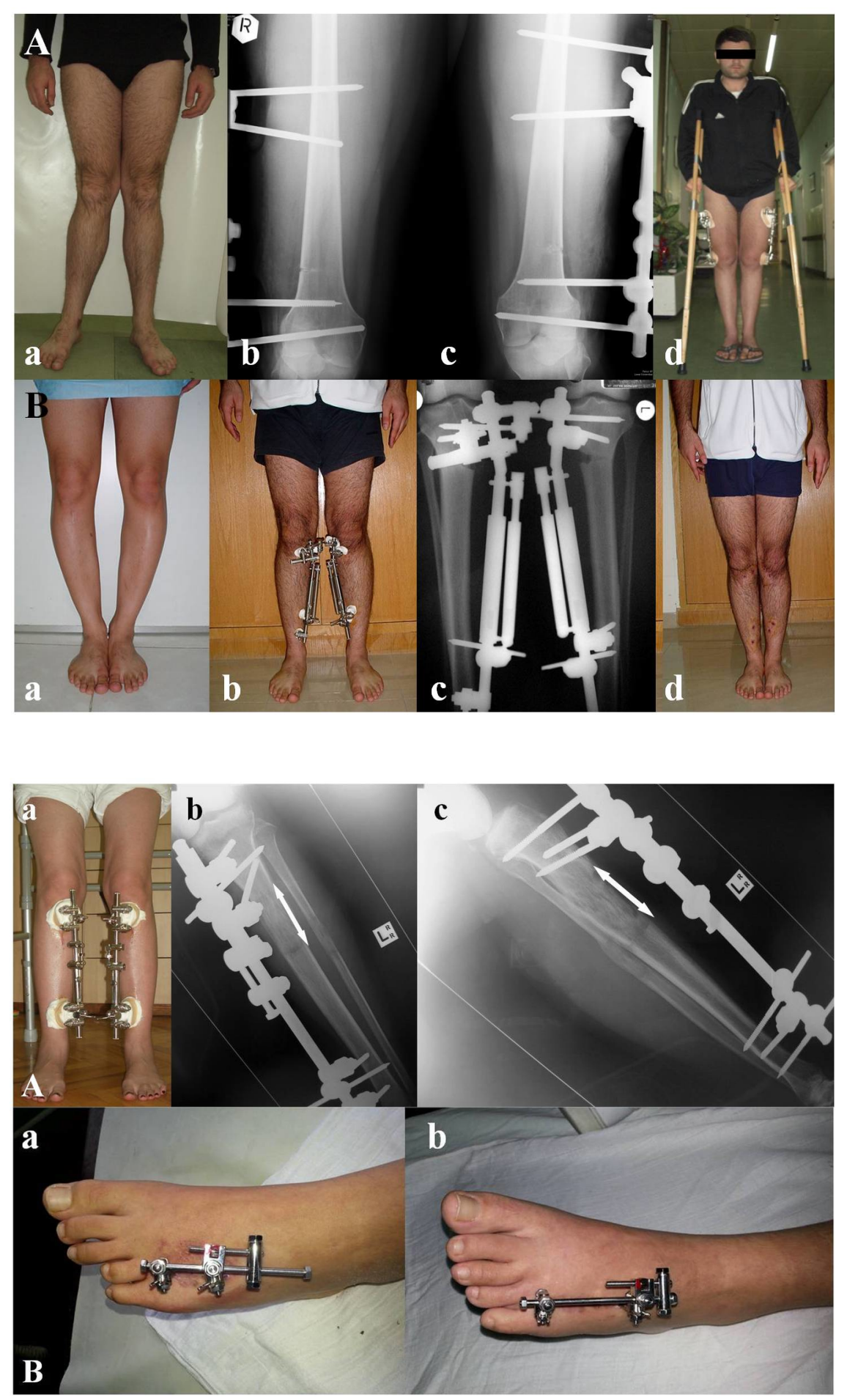